\title{
Effect of Geometric Error of Bearing Parts on the Radial Runout of Rolling Bearing
}

\author{
Yuan-Kun ZHOU ${ }^{1, a,{ }^{*},}$ Yu-Jun XUE ${ }^{1,2, b}$, Zhen-Qiang $\mathrm{CHEN}^{1}$, Yong-Jian YU ${ }^{2}$, \\ Ji-Shun $\mathrm{LI}^{2}$, Wei MA ${ }^{1,2}$ \\ ${ }^{1}$ College of Mechatronics Engineering, Henan University of Science and Technology, Luoyang \\ 471003, China
}

${ }^{2}$ Henan Key Laboratory for Machinery Design and Transmission System, Henan University of Science and Technology, Luoyang 471003, China

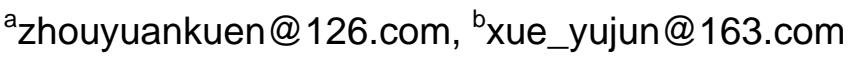

${ }^{*}$ Corresponding author

Keywords: Cylindrical Roller Bearing, Roundness Error, Harmonic Coefficients, Roller Diameter Error, Radial Runout.

\begin{abstract}
According to the movement relationships and geometric relationships of cylindrical roller bearing parts, the mathematical model of the radial runout of bearing outer ring is established. The principle of algorithm for the radial runout of bearing outer ring is analyzed. Numerical simulation of the motion process of bearing is carried out. The effect of outer ring geometric error and rollers diameter error on the radial runout of bearing outer ring is researched. It is found that the radial runout of the bearing outer ring increases linearly with the increase of the outer ring basic circle diameter when the outer ring has the same roundness error. When the outer ring has the same basic circle diameter, the radial runout of the bearing outer ring increases linearly with the increase of second order harmonic coefficients. When the diameter errors of rollers range from $-1.5 \mu \mathrm{m}$ to $1.5 \mu \mathrm{m}$, the maximum runout value of the bearing outer ring is related to the diameters of the two minimum rollers, and the minimum runout value of the bearing outer ring is related to the diameter of the maximum roller.
\end{abstract}

\section{Introduction}

The rotational accuracy of the rolling bearing plays an important role for the rotational performance of equipment shaft, which is a main index for expressing bearing performance. Geometric error of bearing parts is a key factor affecting radial runout of inner and outer rings of bearings. It is significant to study the relationship between the geometric error of bearing parts and the rotation accuracy of bearings.

Okamoto et $\mathrm{al}^{[1]}$.designed a device for measuring radial runout of bearing which is used to analysis the influence law of the shaped error of outer ring raceway and dimension error of the ball, and the distribution of the roller and ball on the rotational accuracy of the bearing. Shi et al. and Song et al. ${ }^{[2-3]}$ developed simulation and prediction model of rotary accuracy of cylindrical roller bearing, and obtained the radial runout curve by simulation and analyzed the relationships between harmonic coefficient, harmonic order of roundness error of raceway and the motion accuracy of bearings. $\mathrm{Li}^{[4-7]}$ presented the new ideas for the detection technology, the evaluation algorithm, the separation and purification method of the cylindricity and roundness error.

This paper established a prediction model on radial runout of bearing outer ring taking cylindrical roller bearing as the research object, in which the roundness error of outer ring and the different roller diameter was considered. It also analyzed the influence laws of the harmonic coefficient of second order roundness error, the base diameter of outer ring raceway and different roller diameter on radial runout of outer ring to support for designing and developing cylindrical roller bearings with high precision. 


\section{The Establishment of Simulation Model}

\section{The Basic Assumptions}

1)The roller and the ring doing planar motion in the radial direction of the bearing. The outer ring has three degrees of freedom, which is the translation of the two vertical direction in the radial plane and the rotation of the outer ring;

2)The inner ring is fixed, the roller and outer ring raceway is not contacting at initial time. There is no relative slide between the roller and the ring;

3)The holder is ideal, and without considering the factors of elastohydrodynamic lubrication and the temperature of bearing.

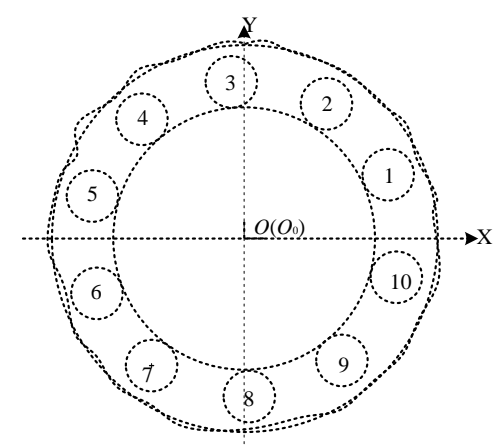

Fig.1 Bearing Initial State

\section{The Simplification of Roundness Error of Outer Ring}

Roundness error of outer ring can be characterized by Fourier series in the polar coordinates.

$$
s(\theta)=\sum_{m=2}^{M}\left[A_{m} \sin (m \theta)+B_{m} \cos (m \theta)\right]
$$

Where $\theta$ is the angle, $s(\theta)$ the radius of vector when angle is $\theta, M$ the highest order of harmonic component of roundness error, $A_{m}$ and $B_{m}$ the sine and cosine coefficients of the $\mathrm{m}$-order harmonic component of the roundness error of the outer ring.

\section{Numerical Simulation Method}

\section{Simulation Method}

In the original location, the ideal center of the inner and outer ring raceway is the origin $O$ of the coordinate system and rollers are distributed on the inner ring raceway uniformly, which is shown in Figure 1. When the bearing rotates, inner ring is fixed; outer ring rotates around the origin of the coordinate system and relative to inner and outer ring, rollers rolls only.

When the outer ring rotates one step, the relative location's changes of outer ring, rollers and inner ring are separated into two steps. Firstly, rollers in the lower location moves to outer ring along the radial direction, that makes rollers in the lower location contact with the outer ring; Secondly, as a whole, the outer ring and rollers move to the inner ring, that makes two rollers at least contact with the inner ring effectively, which is shown in Figure 2. We can get the data of the outer ring's center through the two steps above and then get the radial runout of outer ring of the cylindrical roller bearing by the data processing. 


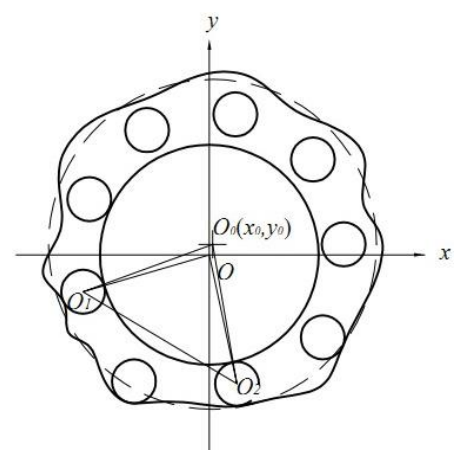

Fig.2 Bearing Stable State

\section{Simulation Verification}

To verify the correctness of the forecasting method of radial runout, and aim at the analytic solutions of radial runout of outer ring which was deduced when the shape of outer raceway is an idea circle. The main parameter is shown in Table.1.

Tab.1 Parameter of Cylindrical Roller Bearing

\begin{tabular}{cc}
\hline parameter & value \\
\hline Diameter of inner & \\
raceway/mm & 50.99 \\
Diameter of outer & 73.03 \\
raceway/mm & 11 \\
Diameter of roller/mm & 14 \\
Number of roller & \\
\hline
\end{tabular}

When all bearing elements are idea circle, the raceway only contact with two rollers below because of the radial clearance of bearing, shown in Fig.3. Select one position in inner ring randomly and infer analytic solution of radial runout of outer ring.

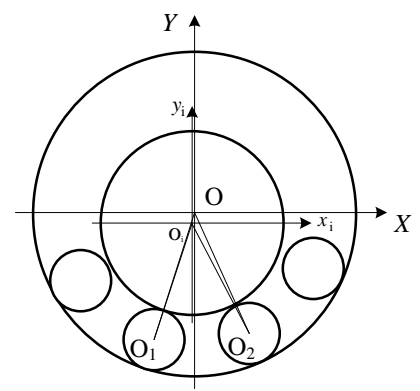

Fig.3 Calculation of Radial Runout When Outer Ring is an Idea Circle

When outer ring turned an angle $\alpha$, the equations can be got from the geometric relation of bearing components:

$$
\begin{aligned}
& O_{1} o_{\mathrm{i}}=\sqrt{\left(X_{\mathrm{i}}-X_{1}\right)^{2}+\left(Y_{\mathrm{i}}-Y_{1}\right)^{2}}=\left(d_{\mathrm{i}}+D_{\mathrm{w}}\right) / 2 \\
& O_{2} o_{\mathrm{i}}=\sqrt{\left(X_{\mathrm{i}}-X_{2}\right)^{2}+\left(Y_{\mathrm{i}}-Y_{2}\right)^{2}}=\left(d_{\mathrm{i}}+D_{\mathrm{w}}\right) / 2
\end{aligned}
$$

Where $X_{1}, Y_{1}, X_{2}, Y_{2}$ means the center coordinate of two rollers at the bottom of bearing, $X_{1}=0.5\left(d_{\mathrm{e}}-D_{\mathrm{w}}\right) \cos \beta_{1}, \quad Y_{1}=0.5\left(d_{\mathrm{e}}-D_{\mathrm{w}}\right) \sin \beta_{1}, \quad X_{2}=0.5\left(d_{\mathrm{e}}-D_{\mathrm{w}}\right) \cos \beta_{2}, \quad Y_{2}=0.5\left(d_{\mathrm{e}}-D_{\mathrm{w}}\right) \sin \beta_{2}$, among them, $\beta$ is the position angle of roller.

Simultaneous equation (2) and (3), the rotation center coordinates can be solved and obtained radial runout of outer ring when it turned an angle $\alpha$.

In order to verify the correctness of the computational model of radial runout in a bearing, and 
gained radial runout of outer ring when outer ring rotated a whole circle, and compared simulation results and theoretical analytical solutions, such as Fig.4, the relative error between them is less than $0.09 \%$. So, the forecasting method which provided in this paper is sufficiently accurate to predict the rotational accuracy of bearing.

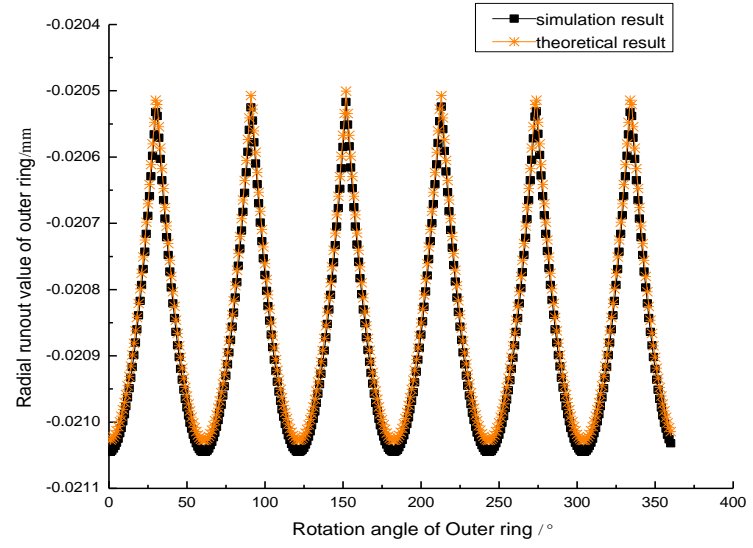

Fig.4 Comparison of Results When the Outer Ring is the Ideal Circle

\section{Effect of the Geometric Error on the Radial Runout of the Outer Ring}

When the harmonic coefficient of the roundness error is $1 \mu \mathrm{m}$, the influence of different ring diameter on the radial runout of the outer ring is shown in Figure 5. Different harmonic coefficient can cause different roundness error, and then the radial runout of outer ring is affected that the influence law is shown in Figure 6 when the base diameter is $75.042 \mathrm{~mm}$.

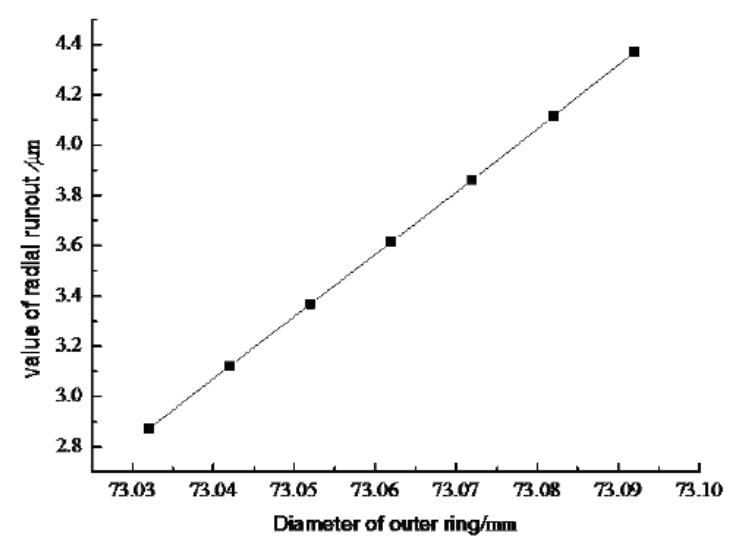

Fig.5 Effect of Diameter of Outer Ring on Radial Runout

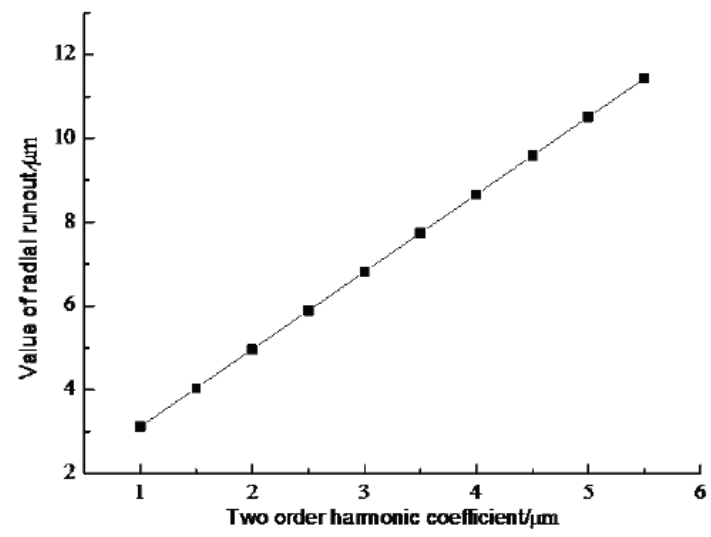

Fig.6 Effect of Harmonic Coefficient on Radial Runout 
The geometric errors of the outer ring raceway can be divided into two parts, the one is base diameter error of outer ring and the other is roundness error of outer ring. At this time the two order harmonic equation $s(\theta)=A_{n} \sin 2 \theta$ is used to show the roundness error of the outer ring, and the profile of outer ring can be expressed $R(\theta)=d_{e}+s(\theta)$. The effects of different $d_{e}$ and different $A_{n}$ on the radial runout of the outer ring are listed in Table 2.

Tab.2 Radial Runout of the Outer Ring with Different Diameter and Roundness Error (Unit: $\mu \mathrm{m})$

\begin{tabular}{c|c|c|c|c|c|c|c}
\hline & & & & & & \\
\hline
\end{tabular}

The simulation results show that the base diameter of the outer ring is changed, which has little influence on the radial runout of the bearing but the roundness error is relatively large, and the radial runout of the outer ring increases linearly with the base circle of the outer ring and harmonic coefficient.

\section{Effect of Different Roller Diameter Errors on the Radial Runout of Outer Ring}

In order to study the influence of roller diameter on the radial runout of outer ring, roller diameter is measured, which error range is $-4 \mu \mathrm{m}-4 \mu \mathrm{m}$. When the effect of the roller diameter on the radial runout of the outer ring is studied, the bearing parameters used in this paper are shown in Table 1.The error of the roller diameter is $\pm 1.5 \mu \mathrm{m}$.

The error values of each roller and the maximum and minimum of radial runout of outer ring are shown in Table 3.Compared to first, second, third groups, the error of the roller diameter is all negative error. In the first group, the third to ninth error of the roller diameter are increasing, the second group are decreasing, and the third group is no error of the roller diameter for third to ninth rollers. Although the roller diameter is different but the maximum and minimum of radial runout of outer ring are the same, there is no effect of other roller of existing errors on the maximum and minimum of radial runout.

With Figure $7 \mathrm{a}$ and $\mathrm{b}$ can be found that the maximum of radial runout of outer ring is determined by the two rollers that diameter error is $-1.5 \mu \mathrm{m}$, and the minimum is determined by rollers of the error free.

Again the error of the roller diameter is all positive error contrast to fourth, fifth, sixth groups. In the fourth group, the second to eighth error of the roller diameter are decreasing, the fifth group are increasing, and the sixth group is no error of the roller diameter for third to ninth rollers. Although the roller diameter is different but the maximum and minimum of radial runout of outer ring are the same, there is no effect of other roller of existing errors on the maximum and minimum of radial runout. 
Tab.3 Radial Runour of the Outer Ring with Different Error of Roller Arrangement

\begin{tabular}{c|c|c|c|c}
\hline $\begin{array}{c}\mathrm{Nu} \\
\mathrm{m}\end{array}$ & Error of roller arrangement $/ \mu \mathrm{m}$ & $y_{0 \mathrm{max}} / \mu \mathrm{m}$ & $y_{0 \mathrm{~min}} / \mu \mathrm{m}$ & $k_{\mathrm{ea}} / \mu \mathrm{m}$ \\
\hline 1 & $-1.5,-1.5,-1.3,-1,1,-0,9,-0.7,-0.5,-0.3,-0.1,0$ & 23.133 & 20.501 & 2.632 \\
\hline 2 & $-1.5,-1.5,-0.1,-0.3,-0.5,-0.7,-0.9,-1.1,-1.3,0$ & 23.133 & 20.501 & 2.632 \\
\hline 3 & $-1.5,-1.5,0,0,0,0,0,0,0,0$ & & & \\
\hline 4 & $1.5,1.3,1.1,0.9,0.7,0.5,0.3,0.1,0,0$ & 21.556 & 19.003 & 2.553 \\
\hline 5 & $1.5,0.1,0.3,0.5,0.7,0.9,1.1,1.3,0,0$ & 21.556 & 19.003 & 2.553 \\
\hline 6 & $1.5,0,0,0,0,0,0,0,0,0$ & 21.556 & 19.003 & 2.553 \\
\hline 7 & $-1.5,-1.5,-1.1,0.3,-0.5,-1.3,0.1,-0.9$, & 23.133 & 19.003 & 4.130 \\
& $0.7,1.5$ & & & \\
\hline
\end{tabular}

With Figure 7c we can be found that the maximum of radial runout of outer ring is determined by rollers of the error free, and the minimum is determined by the two rollers that diameter error is $1.5 \mu \mathrm{m}$. The results suggested that the maximum of radial runout of outer ring is related with the two smallest diameter rollers, and the minimum of radial runout is related with the greatest diameter rollers. Other rollers have no obvious effect on the radial runout of the outer ring.

In the seventh group, the diameter error of third to ninth roller is in disorder, that radial runout process is shown in Figure 7d and validate above presumption.
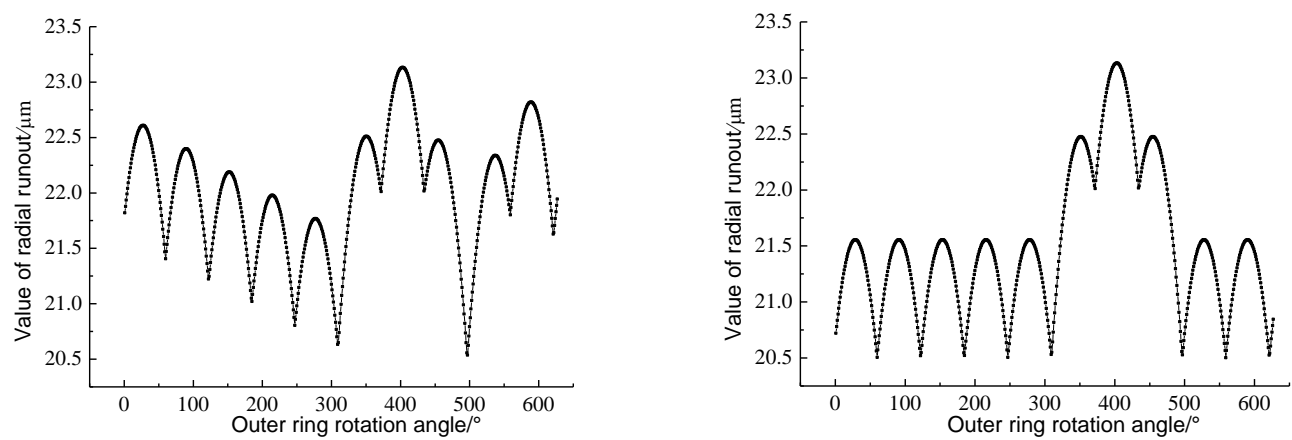

(a) Radial Runout Process with Second Group (b) Radial Runout Process with Third Group
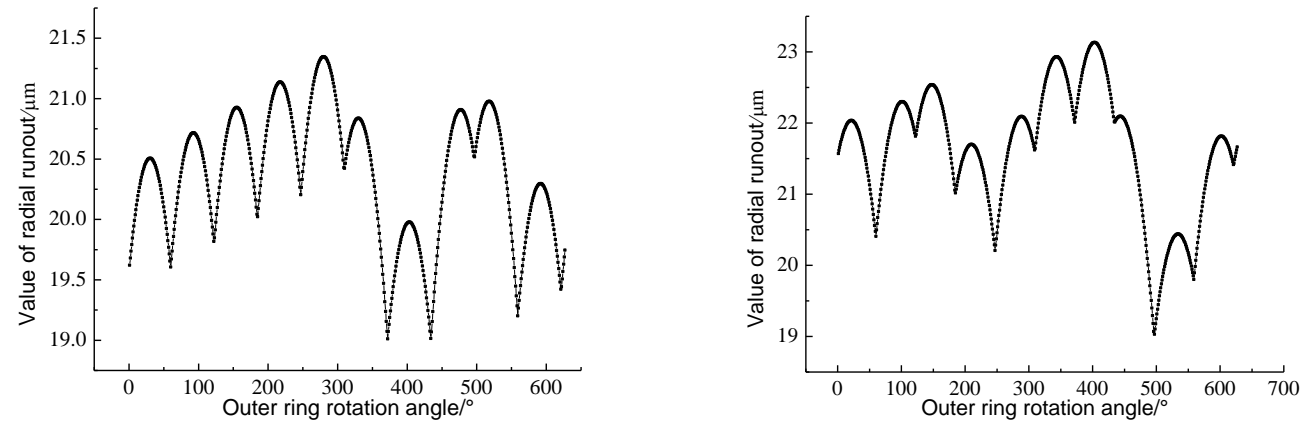

(c) Radial Runout Process with Fifth Group (d) Radial Runout Process with Seventh Group

Fig.7 Radial Runout Process of the Outer Ring with Different Roller Diameters

\section{Conclusion}

(1) When the outer ring raceway is ideal circle and the error of roller diameters in the range of 
$-1.5 \mu \mathrm{m}-1.5 \mu \mathrm{m}$, the maximum of radial runout of outer ring is related with the two smallest diameter rollers, and the minimum of radial runout is related with the greatest diameter rollers. Other rollers have no obvious effect on the radial runout of the outer ring.

(2) The base diameter of the outer ring is changed, which has little influence on the radial runout of but the roundness error is relatively large, and the radial runout of the outer ring increases linearly with the base circle of the outer ring and harmonic coefficient.

\section{Acknowledgement}

The authors gratefully thank the National Natural Science Foundation of China (No. 51375148) and the Project of Basic and Advanced Technology Research of Henan Province of China (No. 142300413217) for financial support. This work is also supported by the Key Research Program of the Higher Education Institutions of Henan Province (No. 15A460022), and the Program for Innovative Research Team (in Science and Technology) in University of Henan Province (No. 15IRTSTHN008).

\section{References}

[1] Junzo Okamoto; Tatsuo Ohmori; Tokio Kitahara. Study on run-out of ball bearings: relation between unroundness of race and locus of shaft in rotation [J]. Japanese Journal of Tribology. 2001(NO.4).

[2] SHI Wenxiang, LI Jishun, LIU Yonggang. Forecast and Simulation of Rotational Accuracy of Cylindrical Roller Bearing [J].Mechanical Science and Technology for Aerospace Engineering, 2011, 45(11): 72-78.

[3] SONG Fei, LI Jishun, LIU Yonggang. Influence of Raceway Roundness Error on Running Accuracy of Cylindrical Roller Bearings [J]. Bearing, 2011(05): 1-4.

[4] LEI Xianqing, LI Jishun, XUE Yunjun, CHANG Weihang. Evaluating Algorithm of Roundness Error Based on Polar-Coordinate Measuring [J]. Journal of Engineering Graphics, 2010(2): 188-191.

[5] LEI Xianqing, LI Yan, LI Jishun, et.al. Measurement techniques of cylindricity with three-point method [J]. Chinese Journal of Scientific Instrument, 2007, 28(5): 944-950.

[6] LEI Xianqing, LI Jishun, LI Yan, et.al. Error Separated Method of Measuring Datum for Cylindricity Measurement [J]. China Mechanical Engineering, 2007, 17(20): 2102-2106.

[7] LI Jishun, LEI Xianqing, XUE Yunjun, DUAN Mingde. Evaluation Algorithm of Cylindricity Error Based on Coordinate Transformation [J]. China Mechanical Engineering, 2009, 20(16): 1893-1897. 